\title{
Protée
}

\section{De « la matière " du visible et des arts}

\section{Marie Renoue}

Volume 36, numéro 3, hiver 2008

Le titre des œuvres : accessoire, complément ou supplément

URI : https://id.erudit.org/iderudit/019638ar

DOI : https://doi.org/10.7202/019638ar

Aller au sommaire du numéro

Éditeur(s)

Département des arts et lettres - Université du Québec à Chicoutimi

ISSN

0300-3523 (imprimé)

1708-2307 (numérique)

Découvrir la revue

Citer cet article

Renoue, M. (2008). De « la matière » du visible et des arts. Protée, 36(3), 99-109. https://doi.org/10.7202/019638ar

\section{Résumé de l'article}

Enjeu de discussions à la fois interdisciplinaires et de traditions diverses, la notion de matière est forcément complexe. Aussi notre texte propose-t-il de considérer la matière au sens commun du terme, celle que l'on décrit lorsque l'on parle du visible et de l'art contemporain en particulier. La question que nous poserons est la suivante : quelle sémiotique peut rendre compte de notre relation perceptive et sensible à la matière visible d'objets d'art ? Pour répondre à cette question, trois oeuvres seront abordées : les Vitraux de Pierre Soulages (1994), les Sculptures et dessins de crin de Pierrette Bloch et les Light Pieces de James Turrell. 


\title{
DE «LA MATIÈRE» DU VISIBLE ET DES ARTS
}

\author{
MARIE RENOUE
}

Rencontrer la matière, c'est manifestement ce que l'on est amené à faire dès que l'on s'intéresse au visible. Et la matière $s^{\prime}$ imposerait d'autant plus aux regards des spectateurs que l'on serait attentif à I'art contemporain, soulignent F. de Méredieu dans son Histoire immatérielle et matérielle de l'art moderne (2004) et le matériologue F. Dagognet traitant de l'exaltation de la matière pour elle-même dans le xxe siècle deTàpies, Dubuffet, Mathieu, Soulages ou Hantaï, tandis que les Anciens l'auraient tenue pour un simple support à effacer au bénéfice de la forme ou de l'idée (Dagognet, 1989: 106).

Cependant, si l'on rencontre la matière sous divers aspects et suivant différentes modalités dans I'histoire de l'art, la définition qu'on en donne implicitement ou explicitement n'est pas sans poser quelques problèmes, même si l'on tente de limiter son point de vue à un domaine d'expression. F. de Méredieu évoque ainsi le renouvellement de la notion scientifique de matière, le décalage du monde des artistes où se juxtaposeraient et se mélangeraient des savoirs venus d'époques et d'horizons différents, ainsi que les enjeux axiologiques de la valorisation de la matière dans un univers épistémologique et religieux qui ne lui était guère favorable (2004: 35). Insistant également sur l'ébranlement de l'axiologie judéo-chrétienne par la mise en valeur de la matière, F. Dagognet analyse les approches divergentes de Hegel, Sartre ou Bachelard, les nouveaux matériaux aux qualités opposées ${ }^{1}$, la « métasubstance moderne » convertible et morphoproductrice qui dérouterait notre savoir expérientiel de la matière (1989: 180-209).

Compte tenu de la diversité et de la complexité de ces définitions, est-il possible de proposer une sémiotique de la matière? Comment l'orienter, quels objets sémiotiques viser dans l'univers discursif de l'art qui nous intéresse? Quelles définitions et quels points de vue la sémiotique, en tant que discipline traitant d'univers de signification, a-t-elle proposés pour aborder la matière? II serait vain de rechercher un point de vue autonome et unique. Comme les autres disciplines, la sémiotique a modifié son approche, plus structuraliste et formaliste dans les années 1970, plus cognitive et sensible dans les années 1980. Si le Dictionnaire de Greimas et Courtés évoque en effet la conception hjelmslévienne de la matière (1979: 223, 368), les écrits plus récents de Greimas, Bastide, Fontanille ou Parret montrent une diversité de points de vue: la matière devenue espace de potentialités signifiantes - plutôt que présupposé abstrait du métalangage descriptif -, horizon visible et sensible en relation directe avec le corps du sujet sémiotique. Le discours des sémioticiens rejoint ainsi les préoccupations et orientations phénoménologiques de plasticiens ou de chercheurs qui, comme A. Joséphine, évoquent un rapport avec la matière «pouvant aller jusqu'à l'intime » et la considèrent «non comme un état de fait [...], mais comme un état de chose à faire» (1997: 34) ou qui, comme M. Collot, traitent de la «matière-émotion» en termes d'intensité et de rythme, «d'appel du sens» (1997: 264, 296 et suiv.).

C'est de cette sémiotisation potentielle de la matière que nous proposons de traiter. Et ce projet invite non seulement à préciser notre objet, à retenir de la vaste méthodologie sémiotique ce qui pourrait s'avérer rentable pour sémiotiser des matières d'art, mais aussi à dessiner une position théorique héritière $d^{\prime}$ 'une tradition sémiotique et capable d'intégrer la diversité des angles d'approches évoqués plus haut. Si, comme nous I'avons souligné ailleurs (Renoue, 2001), il y a une con- 
vergence profonde entre la thèse phénoménologique et les fondements théoriques de la sémiotique, si cette philosophie a par ailleurs influencé les discours tenus sur les objets qui nous intéressent, il conviendra d'affirmer la spécificité d'une approche sémiotique en sémiotisant le phénoménologique. Précisons encore que les objets choisis pour mener cette réflexion - les vitraux de Pierre Soulages, les Sculptures et dessins en crin de Pierrette Bloch et les Light Pieces de James Turrell - ont été sélectionnés pour la diversité des angles de vue qu'ils semblent inviter à parcourir, mais que des traits communs infléchiront leurs modalités d'appréhension. En effet, ressortissant tous du domaine de l'art contemporain, ils présentent également une propension à jouer de la ténuité de la matière, de sa banalité ou de son indétermination. Et cet intérêt pour le ténu motive le type de vision que nous privilégierons, à savoir une vision rapprochée, qui scrute les détails, la texture, plutôt que celle qui, de loin, embrasserait les ensembles, les volumes et les masses. L'attention présupposée ici cherche donc à voir de manière encore plus fine et à décrire ce qui se joue dans l'apparence subtile et changeante de la matière.

\section{Quid des matières et de la sémiotique?}

Quelle matière sémiotiser dans le domaine artistique, suivant le point de vue phénoménologique et perceptif que nous avons esquissé dans notre introduction? Oublier que ce terme garde les traces de conceptualisations antérieures peut paraître peu rigoureux; même la définition courante de la matière - «substance qui constitue les corps, qui est l'objet d'intuition dans l'espace et qui possède une masse mécanique » (Petit Robert) - laisse émerger des traces de théories passées et la préférence du dictionnaire de langue pour une conception moderne et cartésienne face à celle plus dynamique d'Aristote ${ }^{2}$. Il semble, néanmoins, nécessaire de restreindre l'investigation. Ainsi, même si F. de Méredieu évoque une confusion des savoirs et la définition corpusculaire et ondulatoire de la physique contemporaine, elle privilégie dans ses études une approche formaliste ${ }^{3}$ et matiériste ${ }^{4}$ qui motive sa distinction entre le matériel et l'immatériel (2004: 27). Retenons donc seulement une double perspective dynamique et subjective qui apparaît dans nombre d'études contemporaines - et que nous retrouverons en sémiotique. Ainsi F. Dagognet met-il l'accent sur les états mésomorphes et changeants de la matière; J. Petitot, limitant la portée d'une opposition entre phénoménologie des formes et physique de la matière, traite d'auto-organisation ou morphogenèse (dans Constantini et Darrault-Harris, 1996: 169). Les physiciens soulignent l'importance de l'échelle d'observation et $d^{\prime}$ interaction des phénomènes, la relation entre faits observés et fait d'observation ${ }^{5}$ - parfois avec une propension à l'irrationnel d'après G. G. Granger (1990: 227-240) -, tandis que M. Collot, se référant à des "philosophes de la matière sensible» 6 et à Sémiotique des passions, affirme la correspondance affective du sujet au monde, la relation constitutive entre le sens et le sensible et retient comme objets d'analyse le rythme et l'intensité - à partir desquels $C$. Zilberberg a élaboré une sémiotique tensive féconde pour l'analyse du visible et du sensible.

Au sein de cette complexité, où s'est donc situé le discours sémiotique sur la matière? II semble acquis que les premiers auteurs de référence de la sémiotique greimassienne, F. de Saussure et L. Hjelmslev, après avoir défini la matière comme une masse ou un continuum amorphe, l'ont écartée de l'analyse ${ }^{7}$. Comment la postérité sémiotique a-t-elle assumé ce rejet? Dans le Dictionnaire de Greimas et Courtés, la matière apparaît peu, sinon en référence à Hjelmslev comme «le matériau premier grâce auquel une sémiotique, en tant que forme immanente, se trouve manifestée » $(1979$ : 223) ou, dans l'article «substance», comme "support" de signification, pour servir de substance sémiotique» (ibid. : 368). Présentant la forme comme seule analysable, donc comme unique objet du champ de la sémiotique, J. Courtés écarte encore, en 1991, la matière, celle-ci «n'étant que présupposée par l'existence des formes» (1991: 24-25).

Cependant, A. Hénault précise que, si Hjelmslev a retenu « la substance (la convocation du sens) et la forme (I'activité catégorisante) » comme objets d'études potentielles, « aujourd'hui il est difficile de faire l'économie de la perception globalisante du "mening" », sens-matière (1992: 71-72). Deux types de sémiotique peuvent être convoqués pour répondre à cette carence. D'une part, une sémiotique du continu est développée par Greimas et Fontanille (1992) qui, dans le cadre épistémologique ouvert par la phénoménologie, s'appuient sur la conception dynamique du devenir et de la protensivité pour « mettre en scène »l'émergence de proto-sujets et de proto-objets sémiotiques et pour analyser les passions et la perception. J. Fontanille et C. Zilberberg (1998) poursuivront l'étude des émotions et de l'événement, en analysant les valeurs en termes de valences d'intensité et d'extensité, de tempo et de rythme - de quoi façonner une lecture dynamique et énergétique de la matière. D'autre part, en invitant à considérer les choses elles-mêmes, la sémiotique de l'objet a participé au retour de la matière concrète.

Cette sémiotique de l'objet ${ }^{8}$, Greimas l'évoque en 1983 en précisant que les problèmes de l'approbation et de la construc- 
tion des objets semblent, à première vue, se situer à deux niveaux distincts : celui de la perception et celui de la transformation du monde (1983 : 13). Deux études parues en 1987 ressortissent de ce programme bipolaire. L'une répond à la question « comment penser la matière ni scientifiquement, ni philosophiquement, mais telle qu'elle est là [...] sous la forme du monde du sens commun, devant nous », énoncée par Greimas; il s'agit du Traitement de la matière de F. Bastide (1987 : 5). La sémioticienne y considère la matière comme un corps plastique structurellement transformable, une matière-matériau définissable en tant que manières d'être ${ }^{9}$ modulables par l'action déstructurante et restructurante de sujets opérateurs et observateurs attentifs. Par ailleurs, dans son étude de L'Éloge de l'ombre de Tanizaki parue dans De l'imperfection (1987), Greimas distingue une autre démarche à partir de celle évoquée auparavant en spécifiant leurs objets sémiotiques:

1. la matière de l'objet en soi, de l'objet du monde qui est là, rayonnant d'énergie et qui ne touche le sujet qu'accidentellement et 2. l'objet de la perception, présent pour le sujet et éventuellement saisissable par lui. (Greimas, 1987 : 50)

Les matières dessinées par ces discours sur la perception et sur la transformation sont distinctes. Ou il s'agit, avec Greimas, d'une matière sensible, d'un événement esthésique en tant qu'effet de présence énergétique et fugace pour un sujet «ému » et non modalisateur; ou il s'agit d'une matière-matériau transformable et utilisable pour la réalisation d'objets de valeur - d'où les références répétées à la célèbre analyse de « La soupe au pistou » de Greimas (1979). Entre événement esthétique et scrutation de modes d'être ou d'apparaître, entre la matière indéterminée de l'événement et une matière-matériau formellement variable et descriptible, on oscille apparemment entre des conceptions différentes: l'une, plus énergétique, semble ressortir du "divers du phénomène » kantien comme sensation pure ou encore de la description de l'expérience esthésique merleau-pontienne ${ }^{10}$; I'autre serait plus proche d'une matière substantielle moderne et physique. Cette oscillation évoque ce que J. Petitot écrit au sujet de «L'homme à la coquille» de Valéry: «la pensée de l'apparaître morphologique oscille entre recherche $d^{\prime}$ un principe organisateur et évaluation esthétique » (dans Constantini et Darrault-Harris, 1996: 161).

Où situer nos approches de la matière par rapport à ces deux courants? Plutôt que de proposer une troisième voie, c'est une tentative d'englober ces points de vue que nous proposons dans une sémiotique de la matière capable de se déployer suivant les dimensions phénoménologique, formelle et sensible - auxquelles nous limitons pour I'heure nos études de la matière des objets d'art. C'est seulement en tant que la matière peut constituer en art un objet de valeur en soi ou encore l'instance perceptible et sensible d'une sémiose que nous examinerons les œuvres retenues.

\section{Approcher la matière du visible}

Prenant comme point de départ et support de notre analyse des objets visibles plutôt que des discours sur ces objets, nous postulons que les angles d'approche sont orientés par les objets eux-mêmes et par ce que nous en savons ${ }^{11}$. Nous considérons en effet les objets comme " un appel de sens », suivant l'expression de $M$. Collot, ou plus précisément comme un appel de sens potentialisés et convocables par ces objets-là. II ne s'agit donc pas de dire «n'importe quoi » sur un aspect des œuvres en cours de sémiotisation ni de présenter le discours tenu sur l'objet visé comme le seul qui puisse l'être. La question préliminaire est donc celle-ci : quels discours sur la matière les objets permettent-ils de déployer? Quels points de vue peuvent-ils plus ou moins favoriser? Avec les vitraux de Pierre Soulages, ce sont les capacités factitives que nous retiendrons, de même que les états variables et réactifs d'un matériau composite, doté de qualités en partie émergentes et de propriétés translucides complexes. Les Sculptures et dessins en crin de Pierrette Bloch nous amèneront à examiner les tensions sous-jacentes à la forme dessinée ou sculptée par la matière-matériau. Enfin, les Light Pieces de James Turrell, espaces homogènes, opaques et denses de lumière colorée, nous permettront de considérer le caractère paradoxal et multimodal d'une lumière-matière sans forme affectant le corps d'un sujet sensible touché et touchant.

\section{1) Des propriétés et de l'action de la matière:}

les vitraux de Pierre Soulages

Les vitraux de Pierre Soulages sont composés de verres originaux; ils ont été mis au point après de nombreuses recherches et tentatives pour obtenir une qualité de lumière particulière. $C^{\prime}$ est en tant que filtres et transmetteurs de lumière qu'ils seront donc abordés. La sémiotique que nous présenterons est par conséquent une mise en scène narrative et tensive où les propriétés du verre seront regardées comme des modalisateurs informant la lumière solaire.

L'angle de vue adopté est évidemment motivé par la fonction, généralement assignée aux vitraux, de transmettre et de transmuter la lumière solaire en la filtrant et en la contrôlant; il 
I'est également par le projet précis de l'artiste. Reprenant une entrevue de Traverses, F. de Méredieu cite les propos de Pierre Soulages:

J'ai souhaité une translucidité qui ne soit pas produite par un état de la surface du verre comme dans le dépoli, ni ayant l'aspect laiteux de l'opale ou du plaqué opale, mais provenant de la masse même du verre, celle-ci devenant alors émettrice de clarté; [afin d'obtenir ce résultat, il opte finalement pour] un composite fait d'une masse cellulaire de verre, la translucidité provenant de la dévitrification des grains à l'interface des cellules. (Méredieu, 2004: 144)

La translucidité est l'objet de toute I'attention de I'artiste, mais elle implique la non-transparence de la dévitrification. Translucides et non transparents, les vitraux ne laissent en effet rien voir de l'extérieur ou de l'intérieur de l'édifice, ils laissent seulement passer une lumière. La non-transparence est le premier indice visuel de la présence $d^{\prime}$ un corps proche ou distant contre lequel bute le regard, et à ce titre elle intéresse les études de la matière. C'est vraisemblablement la raison pour laquelle F. de Méredieu ouvre son dictionnaire par un chapitre consacré à l'opacité et à la transparence (2004: 55-154). L'historienne y distingue les niveaux suivants: 1 . la transparence pure; 2 . la translucidité qui laisse filtrer la lumière et gomme les figures extérieures; 3. I'opalescence qui « laisserait subsister que la seule aura de la lumière, et comme son tremblement»; 4. I'opacité qui ne laisse rien voir à travers elle (1994 : 21). Si cette échelle mêle passages de la lumière et du regard ${ }^{12}$, elle présente néanmoins l'avantage de marquer les relations de contrariété entre les termes qui nous intéressent - par exemple, la translucidité présuppose unilatéralement la non-transparence.

Quelles sont les qualités ou propriétés qui assurent cette translucidité non transparente? Dévitrifié, le verre a perdu sa transparence; non coloré, il n'influe pas sur la translucidité en modifiant le spectre coloré de la lumière naturelle et, corrélativement, son intensité - comme il en va des vitraux habituels ${ }^{13}$. Ce sont ainsi la masse du verre, sa texture et sa composition qui seront les modalisateurs de la lumière recherchée par Soulages.

De la texture, il faut également noter la bivalence. Sur la face extérieure, le verre est lisse et brillant, semblable à une pellicule réfléchissante et pellucide qui laisserait apparaître par transparence le verre composé sous la surface. À l'intérieur, nul effet de stratification de la profondeur ne s'observe: le verre apparaît légèrement granuleux et irrégulier - un peu à la manière des pierres de calcaire mitoyennes. De la qualité du matériau au traitement de la lumière, des corrélations peuvent être posées à partir des observations précédentes lues comme autant d'indices des opérations en jeu: soit le «lisse » déterminant la brillance et la réflexion de la lumière; le «granuleux » permettant une luminosité douce et témoignant de la transmission de la lumière. Entre les deux, il y a l'épaisseur (5 à 9 mm) du verre composite.

Pour décrire la composition du verre, nous pouvons reprendre les paroles de leur créateur. II s'agit d'opérations complexes allant de la fabrication d'un premier verre - dont la teneur en métaux a été réduite pour éviter la teinte verdâtre des verres ordinaires -, un verre ensuite concassé et dont les morceaux sont répartis dans des moules pour être soumis à une température précise qui, les rendant "gélatineux», leur permet d'adhérer les uns aux autres; la température maintenue puis baissée assure une dévitrification et une cristallisation à l'interface des morceaux rassemblés. Si l'on veut, à la manière de F. Bastide (1987), dégager les opérations élémentaires principales, on pourrait noter entre les deux états du verre obtenus successivement: 1 . le passage du simple au composé - un premier verre homogène obtenu après tri des éléments colorants et un verre final composé de morceaux en partie dévitrifiés -; 2. un retour au compact par adhésion des morceaux ramollis; et 3. une nouvelle structuration du verre stratifié en profondeur. Les deux responsables de cette transformation sont l'outil qui casse et surtout la chaleur intense, stable et durable qui provoque le ramollissement nécessaire à l'agglomération ${ }^{14}$ des morceaux distincts et qui favorise une transmutation du verre partiellement dévitrifié et, ainsi, I'hétérogénéité du matériau verrier initialement homogène.

L'hétérogénéité apparente du verre aggloméré peut être perçue comme la trace du concassage et l'actualisation par la chaleur de modes d'être verrier différents: les changements partiels d'apparence et de propriétés, sous l'effet du feu, d'un matériau homogène et uni. Du point de vue de la transmission de la lumière, cette hétérogénéité complexifie singulièrement les choses, et ce, d'autant plus que les interfaces dévitrifiées des morceaux de verre agglomérés sont plus réfléchissantes. La transmission sera donc modulée suivant la texture et la composition du verre. Ainsi, il est possible d'imaginer que la lumière, non réfléchie à la surface extérieure du verre, pourrait l'être en partie dans la masse épaisse du verre cristallin qui la diffracterait, la détournerait lors de son passage, avant de la laisser sortir (voir figure 1).

Il va sans dire que cette rétention de la lumière dans le verre semble à même de combler les projets de Soulages : freiner la 
Modalisations du passage de la lumière par et dans le verre : réflexion, absorption et transmission partielles

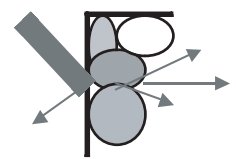

\begin{tabular}{|l|}
\hline Transmission de la lumière de l'extérieur vers l'intérieur \\
\hline passage $\left\{\begin{array}{l}\text { ne pas pouvoir-entrer (réflexion) } \\
\text { ne pas pouvoir-sortir (absorption) } \\
\text { pouvoir-passer (transmission) }\end{array}\right.$ \\
\hline
\end{tabular}

Figure 1

transmission pour donner l'impression que la lumière provient de la masse même du verre, que celle-ci devient émettrice de clarté. La technique principale aura été de répartir des lieux d'impénétrabilité plus ou plus importante au passage de la lumière, et ainsi de la diffracter et de la désintensifier - d'où I'obtention d'une lumière diffuse et non éclatante dans l'espace de l'abbatiale. Nous retrouvons là la sémiotique polémique de la résistance, de la confrontation entre sujets aux dynamiques opposées, à laquelle l'analyse des textes nous a habitués depuis longtemps.

Si le mode d'apparaître de la lumière résulte des opérations en jeu, il témoigne non seulement de l'incapacité de celle-ci à résister aux obstacles matériels, mais aussi de la ténacité de sa dynamique et de sa capacité à se frayer un chemin en se décomposant - une diffraction qui provoque sa désintensification, et donc son amenuisement.

Il convient pour finir de noter la transformation du verre luimême: non seulement de sa luminosité afférente à l'absorption de lumière, mais aussi - et la chose est plus surprenante - de sa coloration. En effet, si le verre présente à l'extérieur les reflets incertains du paysage environnant, il prend à l'intérieur des teintes variées qui rappellent l'extérieur - les vitraux bleus donnent sur le ciel, les roses sur un mur de grès. Ces teintes et ces reflets évoluent avec le temps, apparaissant et s'intensifiant quand la lumière extérieure est importante et indirecte, $\mathrm{s}^{\prime}$ estompant sous l'effet d'une lumière trop intense ou insuffisante. Tout se passe comme si, transformateur de lumière, le verre était à son tour transformé par celle qu'il transforme. L'être coloré des vitraux de Soulages apparaît ainsi comme une qualité émergente modalisée par ses propriétés complexes de transmission-réflexion et par I'intensité de la lumière elle-même - en termes sémiotiques, I'action du verre engendre, sous certaines conditions d'être de l'objet qu'il transforme, son être coloré.
2) De la densité morphodynamique des crins de Bloch

Si les vitraux de Soulages ont offert une occasion pour considérer divers états potentiels de la matière, des modes d'être aux propriétés opposées (la transmission-réflexion du verre en partie dévitrifié) et émergentes, les objets de crin de Pierrette Bloch nous permettront de considérer plus attentivement les relations entre forme et matière. Une citation permet de situer le point de vue de l'artiste dès 1976 :

Pas de point, pas de nœud savant. Rien de ce qui relève d'une virtuosité artisanale, d'un savoir-faire séculaire. Tout cela est loin de mon propos. Cordages, ganses, ficelles sont là pour leur fluidité, leur poids, leur souplesse ou leur fermeté, pour toutes les possibilités formelles qu'elles contiennent en puissance. (Bloch, 1998: 1)

La question que nous poserons est donc la suivante: quelle relation entre la forme et la matière les objets de crin de Pierrette Bloch invitent-ils à voir et qu'en est-il de ces "possibilités formelles » que le matériau contiendrait en puissance?

Produits depuis $1982^{15}$, les objets dont il s'agit sont présentés sur les étiquettes des expositions comme des «Sculptures de crin» ou des «Dessins de crin». Ce qui peut étonner dès l'abord, c'est le «jeu» entre les dénominations génériques et les œuvres - ce dont témoignent le terme pièces et les points d'interrogation attenants aux mots «sculptures» et «dessins» dans le synopsis d'un film sur Pierrette Bloch ${ }^{16}$. Fines, faites de crins noués et enroulés autour d'un fil de pêche fixé contre le mur entre deux pitons, les «Sculptures de crin » sont ténues. La tridimensionnalité de l'objet sculptural est donc réduite au minimum, et la présentation des sculptures fixées à $7 \mathrm{~cm}$ du mur accentue cette impression. Autre objet d'étonnement: à intervalles irréguliers, le crin échappé des boucles et des nœuds tombe, souple et mobile, au moindre courant d'air. Enfin, la banalité du matériau sans grande valeur marchande rattache ces objets à la production contemporaine refusant les matériaux nobles. Dans les «Dessins de crin», les fils de crin bouclés et tombant, fixes et mobiles, ont changé de valeurs; non plus banals et ténus, ils sont originaux, puisqu'ils remplacent, sur des tableaux de bois blanc, les traits immobiles d'encre, de crayon ou de craie.

Comment décrire plus précisément ces objets? Le critère quantitatif est apparu d'emblée évident. Même si les lignes des sculptures peuvent s'étendre sur plusieurs mètres, les formes sont minces. Il y a peu de matière à voir et cette ténuité force à la concentration. Les premières sculptures visibles jouent, de ce point de vue, le rôle de calibreur perceptif et posent une norme moyenne au-delà de laquelle excès et insuffisance 
ont leur place. Dans cette échelle perceptive, le nombre de fils participe à la variation formelle des sculptures dont les fils uniques s'enroulent en vrilles, alors que les fils multiples font un enchevêtrement confus ou forment des boucles. Relativement homogène, en fait, chaque objet présente un rythme régulier et subtil parfois ébranlé par un changement imprévisible. Si les œuvres de crin peuvent donner lieu à une lecture du rythme né de la transformation syntagmatique des formes et de leur mobilité, c'est surtout la torsion du matériau qui retiendra notre attention. Ni pliés ni brisés, les crins forment des courbes plus ou moins concentrées et tendues autour du fil de pêche ou sur la surface des tableaux, et ces tensions diverses témoignent de la densité du crin. Plus précisément, il appert que cette densité du matériau est manifestée par la forme donnée, parce que cette forme est rendue possible par la densité du matériau.

Souligner d'emblée que le crin n'est pas brisé n'indique pas seulement un éventuel « respect» du matériau dont on pourrait percevoir différentes manifestations de la densité; cela souligne aussi que l'effet produit est de l'ordre non pas du discontinu mais bien d'une continuité favorable à l'expression des tensions. Le contraste entre la courbe et l'ondulation des fils qui tombent peut, de ce point de vue, être perçu comme une modulation de la tension. Intensément tendu dans les vrilles et les boucles fines, plus détendu dans les boucles larges, le crin se détend lors de son échappée ondulante sous l'effet de sa propre densité, de sa chute et de son poids. Ce que ces œuvres donneraient à voir, ce serait donc une réaction tensive entre forme et matériau: comment la forme courbée augmente la tension du crin, comment le matériau en chute prend un aspect ondulé en raison de son caractère non pas souple et mou, mais flexible et dense - caractéristiques nécessaires à la courbure des boucles. Ce sont ces corrélations entre formes et tensions apparentes que nous avons représentées dans la figure 2 .

On pourrait encore préciser que la dé-tension du crin libéré de la forme est graduelle et que, près de la boucle, il a une forme raide, encore concentrée et tendue qu'il gardera s'il est court. On pourrait également noter la connivence formelle entre ondulation et boucle, comme si, pour reprendre la célèbre proposition de Focillon, il y avait ici visibilité d'une « vocation formelle » de la matière qui «imposerait sa propre forme à la forme» (1964: 51). La définition aristotélicienne de la substance composée de matière-potentialité et de forme-actualisation montrerait ainsi la pérennité de sa valeur sémiotique. Pierrette Bloch l'avait énoncé dans la citation reproduite plus haut: les matières sont choisies «pour leur fluidité, leur poids, leur souplesse ou leur fermeté,

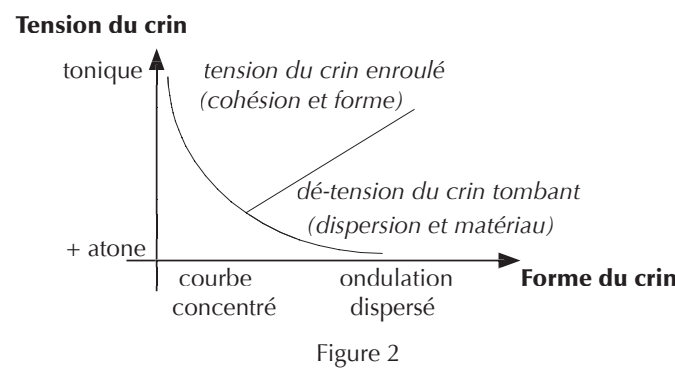

pour toutes les possibilités formelles qu'elles contiennent en puissance». Cette propriété «intentionnelle» du crin, nous I'avons exprimée en termes de densité. Qu'en est-il au juste de cette densité ?

Cité avec des acceptions différentes en sémiotique ${ }^{17}$ ou dans les dictionnaires de langue, le terme densité semble à la fois ressortir, dans les expressions usuelles, de l'intensité (optique dans "la densité des noirs»), de l'extension (l'épaisseur ou le volume d'un «feuillage dense »), du nombre ( «la densité d'une population ») et de la compacité ( «la densité des métaux »). On l'évoque aussi pour signifier le poids et la force. Dans notre description des œuvres de crin, ce sont assurément les valeurs de compacité et de force qui prévalent, ou plus exactement celle d'une compacité du matériau selon qu'elle puisse imposer une dynamique ou une résistance.

Le Petit Robert ouvre sa définition du dense par l'acception suivante: «1. Qui est compact, épais. Brouillard dense $\rightarrow$ impénétrable» (2004 : 687). Si le synonyme impénétrable n'apparaît pas pertinent pour les crins de Pierrette Bloch - compte tenu du point de vue adopté -, il prend une valeur évidente pour les volumes de lumière colorée que sont les Light Pieces de James Turrell.

3) Compacité de la lumière-matière sensible chez James Turrell

Dans la partie de son ouvrage consacrée à l'opacité et à la transparence, F. de Méredieu ouvre un chapitre sur la lumière par une citation de James Turrell :

D'où la lumière vient-elle dans les rêves? Elle est magique, elle a de la substance, elle a une présence physique [...]. J'aime donner de la substance et de la réalité aux choses que nous avons décrétées insubstantielles et transitoires. (Méredieu, 2004: 82)

Le projet et l'objet sont définis: I'artiste américain se propose de jouer du paradoxe en invalidant nos croyances sur la nature insubstantielle de la lumière ${ }^{18}$. Il s'agit de substantialiser la lumière, certes, mais aussi de désubstantialiser l'espace archi- 
tectural et les parois - ainsi que nous pouvons le voir grâce à un article de V. Laganier (2000) illustré par un plan et par des photographies qui montrent la différence entre la « réalité matérielle» du dispositif des Light Pieces et I'apparence de I'installation réalisée à Avignon en 2000. La question que nous nous poserons concerne donc, d'une part, cette conversion des valeurs matiéristes et, d'autre part, l'expérience sensible à laquelle le visiteur des Light Pieces est confronté.

F. de Méredieu le souligne: James Turrell fait partie de ces artistes contemporains qui utilisent l'installation pour plonger le spectateur dans l'image (2004: 597). Il s'agit non pas seulement de rester à distance, de contempler l'œuvre de loin, mais aussi de rentrer dedans, $d^{\prime}$ « habiter» la lumière. Du point de vue du spectateur, que se passe-t-il? Orienté par le dispositif, son parcours le confronte par étapes à des espaces aux valeurs contradictoires. Ainsi, en entrant dans une première salle, il voit face à lui une surface frontale et monochrome, comme un tableau accroché au mur. Tout près, la surface devient poreuse, puis volume illimité de lumière colorée lorsqu'il y pénètre - un volume décrit par G. Didi-Huberman comme « un champ où la lumière est tellement lourde, homogène, intense et sans source, qu'elle deviendra la substance même - compacte et tactile - du lieu tout entier» (2001: 46).

Les termes d'une contradiction perceptive sont posés: on passe de ce qu'on interprète, par habitude picturale, comme une surface dure et pleine, à l'expérience vécue d'une dilution décompacifiante de la surface qui, pénétrable, devient un champ lumineux et compact. Le parcours perceptif est ainsi passages du loin au dedans, de l'interprétation visuelle à l'expérience sensible, de la matière à la dématérialisation de la paroi, de la lumière éclairante et neutre de la première salle à un champ homogène, compact et illimité de lumière colorée et diffuse. Qu'en est-il plus précisément de cette atmosphère lumineuse "compacte» et de la disparition des parois du dispositif final ${ }^{19}$ ?

En fait, c'est de la transparence de la lumière qu'il convient de parler. La lumière n'est pas ici transparente et invisible comme l'éclairage habituel, elle est colorée et, pour ainsi dire, «opaque»- dans le sens où l'on ne voit pas à travers elle ou grâce à elle, mais où on la voit elle seule. La perception est alors ambivalente: cette luminosité opaque noie en quelque sorte les parois, le sol et le plafond et empêche de voir des limites. Elle provoque un espace à la fois ouvert et illimité, mais aussi compact et potentiellement fermé parce que la lumière diffuse et colorée empêche également de voir à distance et qu'elle peut «cacher»-comme il en va du brouillard et de l'obscurité.
Si I'on essaie d'analyser, comme le phénoménologue G. Didi-Huberman, les relations du visiteur-spectateur avec cette atmosphère dans laquelle il peut circuler, nous pouvons relever plusieurs comportements ou sensations. Tout d'abord, notons que le visiteur doit se départir de ses automatismes, de ce que M. Merleau-Ponty appelle les «habitus du corps», le «sens de la situation » qui est une «marge d'existence impersonnelle» (1945 : 94-99). Sans repère dans le champ perceptif, l'orientation et la mobilité sont forcément perturbées. Dans la citation relevée plus tôt, G. Didi-Huberman évoque également la dimension tactile de ces espaces. Non seulement les spectateurs tentent-ils de toucher la lumière qui les englobe 20 mais, d'après le phénoménologue: «C'est à nous toucher que viseraient les œuvres de J. Turrell » (2001: 57 note). La correspondance sensible du visiteur à la lumière diffuse devient également analogique, puisque «l'homme qui marche dans la couleur [...] s'éprouverait lui-même comme devenant flou » (2001: 29).

L'expérience donnée à vivre par cette installation est à plus d'un titre originale. Il ne suffit pas de noter la déconnexion des automatismes déficients de la lecture perceptive du monde et de la mobilité corporelle. II ne suffit pas non plus de déclarer purement et simplement fausse l'interprétation picturale première, puisque de loin le champ lumineux est toujours vu comme un tableau monochrome - quand bien même on viendrait de constater que ce tableau est illusoire. La différence demeure entre la perception distanciée et l'être-dedans du sujet immergé dans une lumière colorée, étrange et profonde ${ }^{21}$. Et, dans ce contexte d'oppositions, le paradoxe est valorisé: I'expérience de l'omniprésence atmosphérique de la lumière et de la couleur remet en question nos habitudes perceptives et l'ontologie du «sens commun » établie par Aristote voyant dans la couleur un accident ou un attribut de la substance. Substantialiser la lumière et la couleur en donnant à les voir comme des phénomènes indépendants de toute surface et indiquer par un dispositif d'oppositions qu'il s'agit de retourner un paradoxe, c'est ce que nous avons retenu des projets de James Turrell.

Traiter de l'illusion, du vrai et des valeurs axiologiques en jeu, la sémiotique s'y est attelée depuis longtemps. Traiter du sensible et du corps, c'est une tâche à laquelle des sémioticiens ont répondu plus récemment en profitant des écrits philosophiques sur le sensible. C'est la raison pour laquelle les analyses de G. Didi-Huberman apparaissent dans des textes comme ceux $d^{\prime} H$. Parret traitant de présence et d'événement (2001). L'importance que ce sémioticien accorde au toucher, à la réversibilité du touchant-touché pour décrire la relation du sujet au monde mérite 
évidemment d'être évoquée pour notre objet, de même que la référence au regard qui «enveloppe, palpe, épouse les choses visibles » du Merleau-Ponty du Visible et de l'Invisible (1964) 22, et la référence à l'œil dépaysé par l'événement de J.-F. Lyotard - un « Il y a » qui «dérangerait I'ordre d'intimité du corps avec lui-même et avec le monde», commente H. Parret (2001 : 24, 53). Les ingrédients relevés dans l'expérience existentielle des Light Pieces sont là : l'événement qui dérange notre perception et désactive notre mode habituel d'être au monde, le rôle du corps dans notre compréhension du monde, la confusion synesthésique du voir et du toucher, la réversibilité du toucher et de l'être touché comme mode relationnel, mais aussi l'isomorphie figurative comme principe de compréhension de «I'homme qui s'éprouverait lui-même comme devenant flou » au sein de cette atmosphère lumineuse.

En 1999, J. Fontanille a abordé en termes plus sémiotiques la question de la mise en discours du sensible et du rôle du corps dans l'émergence de la fonction symbolique. De cette étude des modes du sensible et du corps sémiotique, on peut retenir certains éléments qui permettront d'envisager les distinctions sémiotiques malmenées par l'expérience décrite. Ainsi, citant $\mathrm{H}$. Parret, J. Fontanille envisage le toucher comme une sensation proprioceptive instaurant « l'opposition entre l'identité et l'altérité, [entre] le propre et le non-propre». Ce qui serait en jeu avec le toucher, ce serait «un principe de contact fondamental, qui définirait un champ transitif élémentaire ("la présence pure" et la distinction entre le propre et le non-propre)»... Par ailleurs, la sensori-motricité instaurerait un «champ réflexif », instigateur de la distinction entre le moi et le soi, entre la chair palpitante et I'enveloppe du corps propre (Fontanille, 1999: 30-32). Quant à la vision, débrayée et distanciée, elle "procurerait aux acteurs identifiés une forme, c'est-à-dire une enveloppe spécifique » 23 (ibid. : 44).

Ce que remettraient en cause les Light Pieces, ce seraient donc des modes fondamentaux de constitution des autres, du soi et du non-propre. Dans les termes figuratifs du sémioticien, ce serait l'enveloppe du corps du sujet sémiotique (ibid. : 52) qui perdrait de son pouvoir de résistance aux pressions figuratives de l'extérieur - c'est-à-dire, après la réversibilité des sensations, I'expression d'une confusion figurative entre I'atmosphère diffuse et un corps subjectal devenu poreux. Cette formulation sémiotique, en termes d'enveloppe, de résistance et de pression, évoque évidemment ce que nous avons dit du verre des vitraux de Pierre Soulages, de sa pénétrabilité et de la pénétration de la lumière, d'une sémiotique de la résistance ou de la rencontre entre forces potentiellement opposées. L'intérêt de cette dernière étude réside néanmoins dans la démonstration de la valeur de cette lecture énergétique convoquée pour rendre compte non plus d'une sémiotisation d'un processus physique observable mais de la sémiotisation d'une expérience sensible où la pression, le toucher et le devenir flou sont l'expression d'un sentir dépourvu de corrélat tangible évident.

\section{Conclusion : quelle sémiotique de la matière?}

Nous I'avons évoqué auparavant, notre projet est non pas de nous démarquer de notre école sémiotique, mais plutôt de proposer une démarche englobant les approches subjectales, phénoménologiques et objectales inaugurées par Greimas et Bastide. Les nombreuses références à C. Zilberberg, J. Fontanille ou $\mathrm{H}$. Parret indiquent également ce que nous devons à la sémiotique tensive et à une sémiotique du corporel et de l'esthésie. Mais ces références théoriques et méthodologiques sont accompagnées d'une affirmation toute phénoménologique de la présence matérielle et concrète des choses et des corps, d'un «il y a » résistant qui est « appel de sens», proposition et invitation à une sémiose centrée sur la matière - en tant qu'elle est perceptible, sensible et dynamique. Assumant l'assomption phénoménologique, nous avons ainsi tenté de la sémiotiser pour rendre compte de notre relation et de notre lecture sensible de la matière.

Ainsi que nos études l'ont montré, la sémiotique que nous avons tenté d'élaborer peut se déployer dans trois dimensions: celle des états de la matière - en l'occurrence, des états ou qualités changeants et plus ou moins auto-émergeants -, celle des formes de la matière - et de ses facteurs organisateurs internes ou externes - et celle d'une relation sensible corpsmatière - en l'occurrence d'une relation de contact qui peut s'inscrire dans une mémoire corporelle parfois mise en déroute. Si ce triple déploiement permet de réorienter des réflexions des «matériologues» ou "philosophes de la matière» dans le champ de la sémiotique, s'il présente un intérêt pratique pour décrire et interpréter des objets d'art et, enfin, s'il permet de retrouver les angles d'accroche de la matière des sémioticiens, il a aussi fait émerger à nouveau des questions plus spécifiques: celle, par exemple, de la co-définition des modalités factitives et existentielles - les propriétés dynamiques des vitraux de Soulages où faire et être s'autodéterminent -; celle de la mise en forme comme manifestation de tensions d'origines, de sens et d'intensités différents et plus ou moins quantifiables - la matière des crins de $\mathrm{P}$. Bloch comme potentialité de la forme 
et la forme comme expression de la matière, de son pouvoir de résistance et de réaction -; celle, enfin, de la relation du sujet au monde et de la définition du soi, du même et de l'autre - une question posée à la porosité des enveloppes et aux habitudes d'être des corps.

Précisons encore que l'étude de ces dimensions semble pouvoir convoquer un vaste bagage méthodologique et plusieurs outils conceptuels: la narrativité et les modalités, comme la tensivité et l'aspectualité. Mais une sémiotique de la matière, attentive à la consistance du monde et de ses objets, semble devoir s'attacher plus particulièrement à rendre compte de la densité des choses et des milieux, de la configuration aspectuelle (ouverte-fermée; durable-éphémère) des surfaces et formes et des rapports de forces et de tensions qui travaillent la matière. Autant dire l'ampleur de notre dette à la sémiotique tensive.

\section{NOTES}

1. Dagognet écrit: «la science et l'industrie modifient le concept de matière, dans la mesure où elles parviennent à décider de celle-ci et à la réaliser de telle façon qu'elle puisse inclure des qualités résolument opposées comme le très mince et l'incassable, ou encore le léger et le résistant, etc. » (1989: 187). Les couples évoqués présentent cependant l'inconvénient de relever de catégories sémantiques différentes; ce sont les corrélations entre catégories qui sont révisées, par exemple celle du mince et de l'épais et du cassable et de l'incassable.

2. La distinction aristotélicienne de la matière d'avec la substance composée de matière et de forme ou, en d'autres termes, de potentialité et d'acte, est oubliée du Petit Robert qui définit la matière comme un objet d'intuition, une donnée qui évoque à la fois les sensations affectant le sujet kantien - par opposition à la forme du phénomène qui fait que le divers du phénomène est coordonné dans l'intuition selon certains rapports - et la substance abstraite moderne dont seraient faits les corps, la res extensa divisible, mathématisable et quantifiable qui soutiendra une physique mécanique de corps impénétrables et inertes.

3. L'historienne de l'art ouvre son dictionnaire en ces termes: « L'art est toujours apparu comme la résultante ou la rencontre de deux facteurs opposés, et par voie de conséquence complémentaires, la matière et la forme » (Méredieu, 2004 : 27). Par la suite, elle déclinera cette relation en référence aux œuvres produites et aux commentaires des artistes. Ainsi seront évoquées : 1) les relations d'opposition de la forme contre la matière - dans l'art abstrait en quête de sublimation du matériau -, de la matière contre la forme - avec les œuvres matiéristes au principe d'ordre trop complexe pour pouvoir être saisi -; 2) les relations de correspondance de la matière comme forme - la couleur ou les discontinuités faisant forme - et de la forme comme matière - dans le cas de la répétition défigurante d'une forme.

4. L'historienne présente la matière comme quasi synonyme de maté- riau, mais note que le concept de matière serait plus large que celui de matériau; elle cite Manzini : "comment la matière devient matériau, c'est-à-dire intégrable dans un projet et composante d'un produit» (2004: 28).

5. Dans I'Encylopedia Universalis, J. Guillerme et H. Vérin écrivent: « [...] de nos jours, les formalismes mathématiques qui expriment les vicissitudes de la matière l'identifient à des événements singuliers de lignes d'univers, où nous sommes pris nous-mêmes, sans que nous en ayons conscience ». En ligne: http://www.teilhard.org/panier/1_fichiers/ Trav.La.Matiere_Gr.Ariane.pdf (page consultée le 18 novembre 2008). 6. M. Collot évoque Valéry, Sartre, la «psychanalyse de l'imagination matérielle » de Bachelard, les philosophes des Lumières pour lesquels la sensibilité - affective et sensorielle - serait « conçue comme "propriété générale de la matière" » et les phénoménologues M. Merleau-Ponty et $M$. Dufrenne traitant d'un mode d'être du sujet correspondant à un mode d'être de l'objet (1997: 16-17, 57-59).

7. Dans le Cours de linguistique générale, F. de Saussure définit la substance phonique comme "matière plastique, plan indéfini et indéterminé, [et] masse amorphe» - comme la pensée (1986: 155). J. Fehr précise que, pour Saussure délimitant la sphère du linguistique, "la matérialité - acoustique ou physiologique - [des] "objets extérieurs", telle qu'elle est perceptible par les sens ", est exclue; ce qui importe, c'est «le fait qu'ils servent de signes» (2000: 114). Également écartée de la linguistique formelle de Hjelmslev, la matière ou «mening» est différenciée de la forme et de la substance. Le «mening» danois est traduit tantôt par «matière», tantôt par «sens», en raison, précise $S$. Badir, « de l'absence de terme pour désigner l'in-forme, le non-encoreanalysé, avant la bipartition entre le sensible (de la matière) et l'intelligible (du sens)» (2000: 211). Zone indistincte délimitée de l'extérieur, le «mening» est présenté, par le fondateur de la glossématique, comme un «continuum amorphe et non analysé» (Hjelmslev 1970: 71); il n'aurait d'autre "existence possible que d'être substance d'une forme quelconque» (ibid. : 70). Informe, mais susceptible d'une formation, "le mening " serait de fait inaccessible à toute connaissance puisque non analysable (ibid. : 98).

8. D'après S. E. Larsen proposant une analyse parallèle de la notion d'objet chez Brøndal, Peirce et Greimas, ce dernier se serait tôt opposé à un formalisme relationnel réduisant les objets à des points d'intersections relationnels. Rejetant une conception faisant de la structure sémiotique une pure forme et affirmant l'irréductibilité et la spécificité de la notion d'objet, il aurait rendu possibles une sémiotique de l'objet et une esthétique objectale (1991).

9. F. Bastide dégage des catégories structurelles dans son analyse de la matière. Il s'agit des catégories amorphe versus structuré, discret versus compact, expansé versus concentré, composé versus simple (1987: 11-18).

10. Greimas évoque un «niveau de la sensation pure, des parcelles resplendissant de toutes les couleurs et allant s'introduire dans les yeux » (1987: 51) qui fait écho au texte de M. Merleau-Ponty sur la couleur perçue: «Selon que je fixe un objet ou que je laisse mes yeux diverger ou enfin que je m'abandonne tout entier à l'événement, la même couleur m'apparaît comme couleur superficielle - elle est en un lieu défini de I'espace, elle s'étend sur l'objet - ou bien elle devient couleur atmosphérique et diffuse tout autour de l'objet; ou bien je la sens dans mon œil comme une vibration de mon regard; ou enfin elle communique à tout mon corps une même manière d'être, elle me remplit et ne mérite plus le nom de couleur $\gg(1945: 262)$.

11. La philosopheA. Cauquelin (1992) parle ainsi de textes-objets, C'està-dire d'objets dont la perception est déjà de l'ordre du discursif ou de 
I'interdiscursif - une réactualisation d'autres discours qui, déjà tenus sur les œuvres ou sur l'art en général, en préparent l'appréhension. 12. On peut proposer, pour rendre compte de ces relations, un exercice de modélisation sémiotique (dit 4-groupe de Klein) où les termes seraient considérés comme des complexes définis par les composés: passage du regard permis ou non et passage de la lumière permis ou non. Auquel cas, "passages de la lumière et du regard permis: $A B$ " serait la définition de la transparence; "passage de la lumière seul permis: A et non-B», celle de la translucidité; "passages du regard et de la lumière impossibles: non- $A$ et non- $B$ », celle de l'opacité; quant au "passage du regard seul permis: non- $A$ et $B$ », il est difficile d'en concevoir la possibilité, la lumière étant la condition sine qua non de la visibilité. Tout en montrant la relation de présupposition entre ces deux termes, cet exercice isole "opalescent» dans la gradation du passage de la lumière entre translucidité et opacité.

13. Les couleurs des verres, tout en produisant une coloration des rayons lumineux par tri et absorption d'une partie du spectre coloré de la lumière - ainsi que les physiciens nous l'ont appris -, réduisent I'intensité de la lumière transmise. E. Viollet le Duc (1980: 146), puis L. Grodecki (1954 : 187-191, 202) ont ainsi étudié et relevé le pouvoir de transmission différent de certaines couleurs translucides des vitraux anciens. Ainsi, le verre violet se présenterait comme extrêmement rayonnant, certains bleus et le blanc très lumineux seraient utilisés pour éclairer l'espace, alors que le verre rouge constituerait un écran optique qui deviendrait noir lorsque le soleil ne le frapperait pas directement. 14. L'agglomération est définie comme un tout dont les parties sont liées, dans l'esquisse d'une ontologie matérielle de J.-F. Bordron qui, adoptant le point de vue de l'intentionnalité eidétique et de la méréologie, analyse les différents types relationnels (1991: 59).

15. Lors d'un entretien, P. Encrevé et Pierrette Bloch évoquent la variété de son œuvre: ses collages, ses peintures et dessins, et le travail du crin de cheval - ses mailles de crin succédant à ses mailles de chanvre, de lin ou de filin goudronné en 1979, I'utilisation des fils de crin à partir de 1982 pour les sculptures et les dessins. Le matériau y est mis en valeur pour sa ténuité, sa longueur et l'ombre projetée (Bloch, 1998: 5-22). 16. Voir la présentation du film de Thierry Spitzer (1997).

17. Le terme densité apparait dans plusieurs textes de sémiotique. Citonsen deux. Dans "Catégories, icônes et types phénoménologiques », J.-F. Bordron traite de la mise en forme d'un continuum plus ou moins amorphe qu'il présente comme l'a priori matériel de la perception tendu entre extensité et atomicité. II distingue ainsi trois catégories principales qu'il analyse successivement: 1) la matière (ou quantité) exfoliée en densité, disposition (texture, souplesse, ...) et force; 2) la qualité analysée en dominante, saturation et intensité; et 3) la forme (ou relation) analysée en extension, limite et direction. Et l'auteur de conclure: "On aimerait dire que ce déploiement catégorial, au sens où nous avons interprété cette notion, assure une communauté des sensations puisqu'il établit une phénoménalité en laquelle toute sensation peut se reconnaître" (2000: 16-17). J. Fontanille et C. Zilberberg évoquent une densité plus générale: "la densité existentielle ou de présence » qui mesurerait des degrés entre modalités de présence et d'absence (1998: 98-99).

18. F. Bastide évoque des objets immatériels, «objets faits de pure lumière» ou bien objets cognitifs (1987: 23).

19. Rappelons, à la suite de C. Adcock, qu'un phénomène optique de " champ total » ou Ganzfeld a été décrit parW. Metzger en 1930. Celui-ci aurait noté que des observateurs, incapables de voir la structure fine d'une surface courbe lorsque l'éclairage est réduit, perçoivent un brouillard de lumière. Reprenant les expériences dans les années 1950-1960, J. J. Gibson décrit: "What my observers and I saw under these conditions could better be described as "nothing" in the sense of "no thing". It was like looking at the sky. There was no surface and no object at any distance. Depth was not present in the experience but missing in it. What the observer saw, as I would now put it, was an empty medium »(Gibson cité par Adcock, 1990). (Notre traduction: «Ce que mes observateurs et moi avons vu dans ces conditions pourrait mieux être décrit comme: "rien" dans le sens de "aucune chose". C'était comme regarder le ciel. II n'y avait ni surface ni objet à aucune distance. La profondeur n'était pas présente dans l'expérience, mais elle manquait en elle. Ce que l'observateur voyait, ainsi que je pourrais maintenant le formuler, était un médium vide»).

Plusieurs ouvrages traitent des effets visuels de dématérialisation par la lumière. C. Zilberberg analyse ainsi les relations entre rayonnement et matérialité dans le baroque et le classique d'H. Wölfflin (1992: 46). L'architecte $\mathrm{H}$. Ciriani décrit une lumière picturale essayant de dégager I'espace de la gravité, de tirer la matière vers l'abstraction (1991 : 82-83.) Sur la lumière diffuse, cf. Renoue 2001.

20. Notre lecture peut être enrichie par deux citations relevées en 2001 sur des sites Internet aujourd'hui appauvris. James Turrell aurait déclaré à J. Brown lors d'une entrevue: "I'm interested in the weights, pressures and felling of the light inhabiting space itself and in seeing this atmosphere $[\ldots]$ atmosphere is volume, but it is within volume $[\ldots]$ there are densities and structuring within a space, and the light has a quality of tangible presence, $[\ldots]$ it has a quality seemingly intangible, yet it is physically felt. Often people reach out to try to touch it » (Interview with James Turrell: http://www.rodencrater.org/seeing/intrview/01. $\mathrm{htm}$ ). (Notre traduction: "Je m'intéresse aux poids, aux pressions et à la sensation de la lumière occupant l'espace lui-même et à la saisie de cette atmosphère $[\ldots]$ I'atmosphère est volume, mais elle est dans les limites d'un volume [...] il y a des densités et des éléments structurants à l'intérieur de ce volume, et la lumière a une qualité de présence tangible, [...] elle a une qualité apparemment intangible, pourtant elle est physiquement ressentie. Souvent les gens ont tendance à essayer de la toucher $»$ ).

21. Cette profondeur peut évoquer celle, non géométrique, décrite par M. Merleau-Ponty : « une profondeur primordiale qui, [sous la profondeur objectivée, serait] l'épaisseur d'un médium sans chose [et non la relation] entre des choses ou même entre des plans, détachée de l'expérience et transformée en largeur. [...] La profondeur ainsi comprise est plutôt l'expérience de la réversibilité des dimensions, d'une "localité" globale où tout est à la fois, dont hauteur, largeur et distance sont abstraites, d'une voluminosité qu'on exprime d'un mot en disant qu'une chose est là » (1999: 65).

22. Après avoir analysé l'exploration de la main et les mouvements du regard, M. Merleau-Ponty écrit: " [...] le spectacle visible appartient au toucher ni plus ni moins que les "qualités tactiles". Il faut nous habituer à penser que tout visible est taillé dans le tangible, tout être tacite promis en quelque sorte à la visibilité, et qu'il y a empiétement, enjambement, non seulement entre le touché et le touchant, mais aussi entre le tangible et le visible qui est incrusté en lui, comme, inversement, lui-même n'est pas un néant de visibilité, n'est pas sans existence visuelle » (1999: 175). Pour un aperçu historique des études comparatives en psychologie et philosophie des modalités sensorielles d'ordre visuel et tactile, voir J. Lupien (2000).

23. "Pour identifier la forme de l'autre corps, la vue apprécierait la zone de contact entre la lumière (l'actant source de la vision) et les obstacles matériels qui occupent le champ (les actants de contrôle de la vision)», précise J. Fontanille (1999: 43). Les modes de manifestation de cette zone de contact - ou de conflit puisque la lumière traverse ou 
ne traverse pas l'obstacle - ont été étudiés dans Sémiotique du visible (1995). À côté des lumières informant éclat, éclairage et chromatisme, retenons la description sémiotique d'une lumière-matière et d'effets de sens-matière décrits comme la manifestation d'un actant « rayonnement » et d'une forme d'occupation de l'espace inaugurant le mode tactile d'appréhension du monde (Fontanille, 1995 : 34-37).

\section{RÉFÉRENCES BIBLIOGRAPHIQUES}

ADCOCK, C. [1990] : James Turrell. The Art of Light and Space, BerkeleyLos Angeles, University of California Press.

BADIR, S. [2000] : Hjelmslev, Paris, Les Belles Lettres.

BASTIDE, F. [1987] : "Le traitement de la matière», Actes Sémiotiques. Documents, vol. Ix, no 89 (numéro complet), Paris, EHESS.

BLOCH, P. [1998] : Pierrette Bloch. Sculptures et dessins de crin, collages, 1968-1998 (catalogue) - entretien avec P. Encrevé: "L'ombre de I'écriture »-, Cajarc (Lot), Maison des Arts Georges-Pompidou.

BORDRON, J.-F. [1991]: «Les objets en parties», Langages, no 103, 51-65.

Visio, vol. 5, no 1, 9-18.

Cauquelin, A. [1992]: L'Art contemporain, Paris, PUF, coll. "Que sais-je?».

CIRIANI, H. [1991] : "Tableau des clartés », Architecture d'Aujourd'hui, no $274,82-83$.

COLLOT, M. [1997] : La Matière-émotion, Paris, PUF.

Constantini, M. et I. Darrault-Harris [1996] : Sémiotique, phénoménologie, discours, Paris, L'Harmattan.

Coquet, J.-C. et J. Petitot (dir.) [1991] : «L'objet et sa réalité », Langages, no 103, numéro complet.

COURTÉs, J. [1991] : Introduction à la sémiotique narrative et discursive, Paris, Hachette.

DAGOGNet, F. [1989] : Corps réfléchis, Paris, Odile Jacob.

DIDI-HUBERMAN, G. [2001]: L'homme qui marchait dans la couleur, Paris, Minuit.

FEHR, J. [(1997) 2000] : Saussure entre linguistique et sémiologie, trad. de P. Caussat, Paris, PUF.

FOCILLON, H. [(1934) 1964] : La Vie des formes, Paris, PUF.

FONTANILLE, J. [1995] : Sémiotique du visible, Paris, PUF; [1999] : «Modes du sensible et syntaxe figurative », Nouveaux Actes Sémiotiques, no61-62-63 (numéro complet), Limoges, PULIM. FONTANILLE, J. et C. ZILBERBERG [1998] : Tension et Signification, Liège, Mardaga.

Granger, G. G. [1990] : L'Irrationnel, Paris, Odile Jacob. GreIMAS, A. J. [1983] : Du sens II, Paris, Seuil; [1987] : De l'imperfection, Périgueux, Fanlac.
GreIMAS, A. J. et J. COURTÉS [1979 et 1986] : Sémiotique. Dictionnaire raisonné de la théorie du langage, 2 tomes, Paris, Hachette.

Greimas, A. J. et J. Fontanille [1991] : Sémiotique des passions, Paris, Seuil.

GRODECKI, L. [1954]: "La couleur dans le vitrail du XIIe au XVIe siècle », dans I. Meyerson (dir.), Problèmes de la couleur, Paris, SEVPEN, 183-205.

HÉNAULT, A. [1992] : Histoire de la sémiotique, Paris, PUF.

HJelmsLeV, L. [(1943) 1970] : Prolégomènes à une théorie du langage, Paris, Minuit.

JOSÉPHINE, A. [1997] : «Critique du visible», Recherches en esthétique, no3 (La Critique), revue du CEREAP, 31-34.

LAGANIER, V. [2000]: "James Turrell: Space division constructions", Actualités de la scénographie, no 113, 16-21.

LALANDE, A. [(1921) 1992]: Vocabulaire technique et critique de la philosophie, Paris, PUF.

LARSEN, S.E. [1991]: "Un essai de sémiotique transatlantique: la notion d'objet chez Brøndal, Peirce et Greimas », Langages, vol. 25, no 103, 7-22.

LUPIEN, J. [2000]: "Tactilité et représentation identitaire dans I'art contemporain », Visio, vol. 5, no 1, 55-66.

MÉREDIEU, F. de [2004]: Histoire matérielle et immatérielle de l'art moderne, Paris, Larousse.

Merleau-Ponty, M. [1945] : Phénoménologie de la perception, Paris, Gallimard;

[(1964) 1999] : Le Visible et I'Invisible, Paris, Gallimard;

Seuil.

PArret, H. [2001] : "Présences », Nouveaux Actes Sémiotiques, n7677-78, Limoges, PULIM, numéro complet.

Renoue, M. [2001] : Sémiotique et Perception esthétique, Limoges, PULIM;

— [2002]: «De la description sémiotique. Une place pour la densité? », Bulletin de l'Association Française de Sémiotique, vol.2, Limoges, PULIM, 32-39.

SAUSSURE, F. de [(1916) 1986]: Cours de linguistique générale, texte établi par C. Bailly et A. Sechehaye, édition préparée et annotée par T. de Mauro, Paris, Payot.

SPITZER, T. [1997] : Pierrette Bloch, boucles, film documentaire, Paris, Arkadin production, 26 min., couleur.

VIOLLET LE DUC, E. I(1855) 1980] : Encyclopédie médiévale, Paris, Interlivres.

ZilberberG, C. [1992] : "Présence de Wölfflin », Nouveaux Actes Sémiotiques, no23-24 (numéro complet), Limoges, PULIM;

$111-143$. 